\title{
On the near field behavior of inclined negatively buoyant jets
}

\author{
L.A. Besalduch ${ }^{\mathrm{a} 1}$, M.G. Badas $^{1}$, S. Ferrari ${ }^{1}$, and G. Querzoli ${ }^{1}$ \\ ${ }^{1}$ University of Cagliari, DICAAR (Dipartimento di Ingegneria Civile, Ambientale e Architettura), 09123 Cagliari, Italy
}

\begin{abstract}
We have performed an experimental campaign in the laboratory on the near field behavior of inclined negatively buoyant jets, issuing from a circular sharp-edged orifice, in order to investigate the symmetry properties of this phenomenon. The velocity measurements were obtained via a non intrusive image analysis technique, namely Feature Tracking Velocimetry. We present here both first and second order statistics, showing that the asymmetry of inclined negatively buoyant jets cannot be considered only a far field feature of this phenomenon, as it arises very close to the release point.
\end{abstract}

\section{Introduction}

The main target of this work is the investigation of the near field features of inclined negatively buoyant jets (INBJs) issuing from a sharp-edged orifice.

When a fluid is released upwards into a lighter receiving fluid or downwards into a heavier one, the physical phenomenon that develops is called negatively buoyant jet. As far as the release is vertical, this is a deeply investigated symmetric phenomenon but, as soon as the release has a different inclination, the misalignment between the initial momentum and the buoyancy determines a much more complex phenomenon.

The previous investigations on INBJs available in scientific literature (e.g. Roberts et al. 1997 [1] and List et al. 1979 [2]) have highlighted a symmetric simplejet-like behavior, near to the origin, followed by an asymmetric one, with a larger widening of the lower region compared to the upper one. Conversely, from some previous investigations carried out in our laboratories (Besalduch et al. 2013 [3]), with more practical targets, the second order statics of the velocity field has shown that this lack in symmetry arises very close to the origin. In order to deeply investigate this apparent contradiction, we have performed a new experimental campaign focusing on the near field behavior of inclined negatively buoyant jets. In this work, the near field of an INBJ is defined as the region between the origin and before the point of maximum height, as in this point the interaction between the upward branch and the downward one deeply affects the physics of the phenomenon, highly complicating the study.

There are many practical applications: of INBJs: sea discharges of effluents from wastewater treatment plants (Koh and Brooks 1975 [4), exit snow from snow ${ }^{a}$ Corresponding author: besalduch@unica.it ploughs (Lindberg and Petersen 1991 [5]), sand and slurry jets as dredging and island building operations and pulverized coal combustion (Hall et al 2010 [6]). One of the most important applications are certainly sea discharge of brine from desalination plants through submerged outfalls, e.g. Ferrari et al 2010 [7], Cipollina et al. 2005 [8]. due to large diffusion of this kind of plants, made to cope the increase of water request in particular in arid region.

In this work INBJs were emitted from a sharp edged orifice; this kind of release, although being a common operative configuration in discharge since it, has been thoroughly investigated in literature with respect to the ones released at the end of a long pipe or with a smooth contraction, moreover the former configuration determines more complex phenomena than the latter, for example due to the inward radial component of the velocity, that is apparent in the well known vena contracta, or the unsteadiness due to the upstream separation, Mi et al. 2001 [9], 2007 [10].

\section{Experiments}

The laboratory section was set-up in order to reproduce a standard configuration of sea discharge, i.e. a portion of pipe, laid down on the sea floor, which discharges the effluent from the orifices along its wall (Wright et al. 1982 [11], Avanzini et al. 2006 [12]). The adopted set-up, described in more details in Besalduch et al. 2013 [3], consists in a $21 \mathrm{~m}$ long and $30 \mathrm{~cm}$ wide flume, with glass walls, filled with $46 \mathrm{~cm}$ of water where the discharge comes through a pipe, which is connected to a constant head tank, by means of a cylindrical vessel of $0.10 \mathrm{~m}$ diameter, with a sharp-edge orifice of diameter $\mathrm{D}=4 \mathrm{~mm}$, on its lateral wall. The released fluid is a solution of water, sodium sulphate and pollen particles, 
to perform experiments with a Feature Tracking Velocimetry (FTV) technique (see below). The jet middle vertical section is lighted by a light sheet, generated by a diode pumped green laser. The phenomenon is recorded by a high speed video camera.

The non-dimensional parameters that control the behavior of INBJs are the Reynolds number Re, the densimetric Froude number Fr, and the angle to the horizontal $\theta$. Re is the ratio of inertial to viscous forces:

$$
R e=\frac{U_{0} D}{v}
$$

where $U_{0}$ is the mean velocity at the outlet, $D$ is the diameter of the orifice, $v$ is the kinematic viscosity of the discharged fluid,

Fr is the ratio of inertial to buoyancy forces:

$$
F r=\frac{U_{0}}{\sqrt{g \cdot \frac{\Delta \rho}{\rho} \cdot D}}
$$

where $g$ is the gravitational acceleration, $\rho$ is the density of the receptor and $\Delta \rho$ is the difference in density between the discharged fluid and the surrounding fluid. $\theta$ controls the misalignment between the buoyancy and the initial momentum.

The values of these parameters in the present experimental campaign are $\mathrm{Re}=1000$ (higher than the critical Re for the apparatus), $\mathrm{Fr}=8$ and $15, \theta=65^{\circ}$.

A Feature Tracking Velocimetry algorithm was adopted to measure velocity fields in a non intrusive way. As better described in Besalduch et al. [12], the main ideas of this technique are:

- to compare windows only where the motion detection may be successful, that is where high luminosity gradients, are identified using the Harris corner detection (a corner is a region with high luminosity gradients along the $\mathrm{x}$ and y direction) (Harris and Stephens, 1988 [13]);

- to measure the displacement as the one minimizing the dissimilarity computed using the Lorentzian estimator, which is a robust (i.e. insensitive to outliers) estimator;

- to validate the samples via an algorithm based on a Gaussian filtering of first neighbors (defined by the Delaunay triangulation).

As a consequence, this technique is able to perform measurements on a wide range of seeding density and in presence of non-homogeneous seedings, where the commonly adopted techniques can be affected by significant errors.

\section{Results and discussion}

In order to show the general behavior of an INBJ, in Figure 1 the mean velocity field $U$, normalized by the maximum velocity at the outlet $\mathrm{U}_{\max }$, for an INBJ with $\mathrm{Fr}=8, \operatorname{Re}=1000$, and $\theta=65^{\circ}$ is shown. The jet axis, defined as the locus of maximum intensity velocity on sections orthogonal to the jet, is superimposed to the velocity field as a dash-dotted black line. The INBJ is driven by two forces acting in different directions: momentum and buoyancy. Figure 1 shows how the INBJ covers a very short initial distance, where it is driven essentially by the initial momentum and maintains a width similar to the diameter of the orifice while, after this distance, the onset of the KelvinHelmholtz billows causes the jet widening. As the distance from the orifice increases, the buoyancy becomes more and more relevant, finally bending the jet downwards. After this point, where the jet reaches its maximum height, the INBJ become similar to a plume in its descending branch. Looking at Figure 1, it is possible to note that the descending branch experiences a sudden widening at $\mathrm{Y} / \mathrm{D} \cong 10$; this is due to the interaction between the upward branch and the downward one, as the mean velocities in the upward branch tend to balance with the ones in the descending branch, so preventing the widening of this last one. As already stated in the introduction, we here focus on the symmetry properties of the near field of INBJs, defined as the region between the origin and before the point of maximum height, where the interaction between the upward and downward branches strongly complicate the physics of the phenomenon.

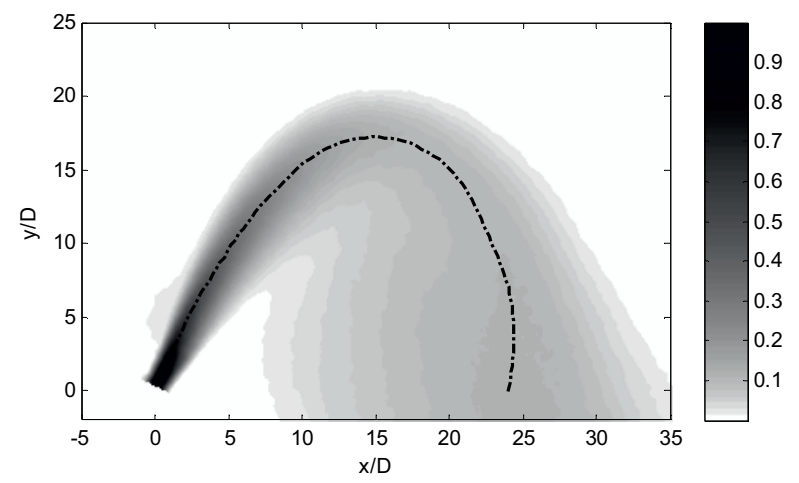

Figure 1. Map of the non-dimensional mean velocity $\mathrm{U} / \mathrm{U}_{\max }\left(\mathrm{U}_{\max }\right.$ is the maximum velocity at the outlet) for an INBJ with $\operatorname{Re}=1000, \mathrm{Fr}=8, \theta=65^{\circ}$; the dashdotted black line is the jet axis (defined as the locus of maximum intensity velocity)

Figure 2 shows the mean velocity field $U$, normalized by $U_{\max }$, in the near field of two INBJs with $\mathrm{Re}=1000$, $\theta=65^{\circ}$ and two different Fr: $\mathrm{Fr}=8$ and 15 (Figure 2a and $2 \mathrm{~b}$ respectively). In the plots, the jet axis is displayed as a dash-dotted black line. The two plots show a compact jet core near the jet origin, while at few diameters from the orifice the different stratifications in the upper and lower region of the jet (stable the former and unstable the latter) cause an apparent asymmetric development of the INBJs, which widen more in the lower region, where the evolution of the KelvinHelmholtz billows is favored by the unstable stratification. From another point of view, it is possible to state that the velocity core and, consequently, the INBJ axis tend to be closer to the upper boundary of the jet than to the lower one, as the detachment of descending plumes tends to erode the velocity core more in the lower region than in the upper one. This 
lack of symmetry is enhanced for the heavier jet $(\mathrm{Fr}=8)$, whose axis bends at shorter non-dimensional distances, s/D, from the origin with respect to the lighter one $(\mathrm{Fr}=15),(\mathrm{s} / \mathrm{D}$ is the abscissa measured streamwise along the axis from the origin).
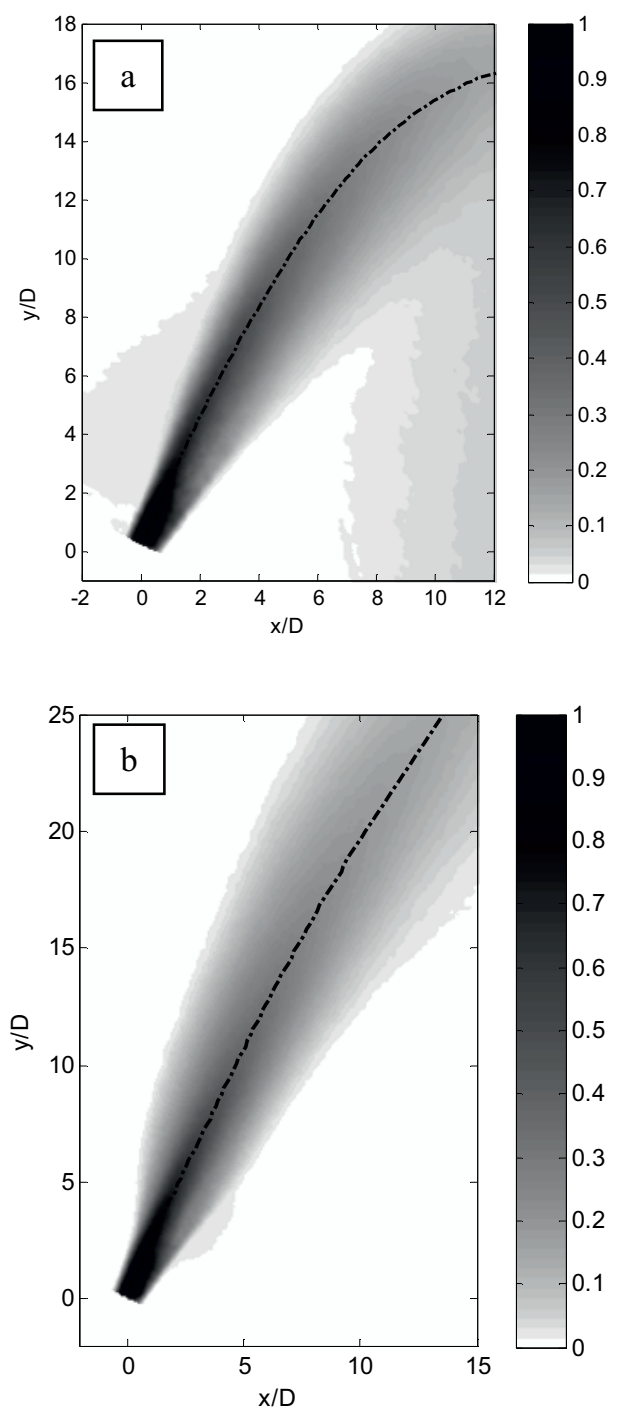

Figure 2. Map of the non-dimensional mean velocity $\mathrm{U} / \mathrm{U}_{\max }$ in the near-field of an INBJ with $\mathrm{Re}=1000$, $\theta=65^{\circ}, \mathrm{Fr}=8$ (a) and 15 (b); the dash-dotted black line is the jet axis.

In order to highlight the role of the densimetric Froude number Fr in the behavior of INBJs, in Figure 3, the widening of two INBJs with $\operatorname{Re}=1000, \theta=65^{\circ}$ and two different Fr ( 8 in red and 15 in blue) is measured along the axis and plotted, versus $\mathrm{s} / \mathrm{D}$, together with the simple jet data $(\mathrm{Fr}=\infty)$ by Quinn 2006 [14], issuing from a sharp edged orifice (black dots) and from a contoured nozzle (black crosses). The widening is defined, for each $\mathrm{s} / \mathrm{D}$, as the orthogonal to the axis nondimensional distance $r_{1 / 2} / D$ between the two points, in the upper and lower region, where the velocity assumes a value which is half of the axial velocity.

This plot highlights the role of the buoyancy, as the Reynolds number is the same for the two INBJs. It is noticeable that both the INBJs widens more than simple jets and that their widening rate after the initial stage (from $\mathrm{s} / \mathrm{D} \cong 6$ on) is higher than the simple jets ones. In order to measure this widening rate, which is relevant as it is proportional to the entrainment and so to the dilution, the data for each case, starting from the point where the widening rate becomes substantially constant, have been fitted, in a least mean square sense, with a straight line. It is possible to note that the widening rate, measured by the inclination $\mathrm{m}$ of the straight line, of the INBJS is larger than the one of the simple jets. Moreover, the widening rate of the lightest INBJ is higher than the one of the heavier INBJ, highlighting the role of Fr in the widening: as Fr decreases (and so buoyancy increases), the buoyancy tends to prevail on the momentum and INBJs tend to remain confined in a smaller space, so experiencing a lower widening.

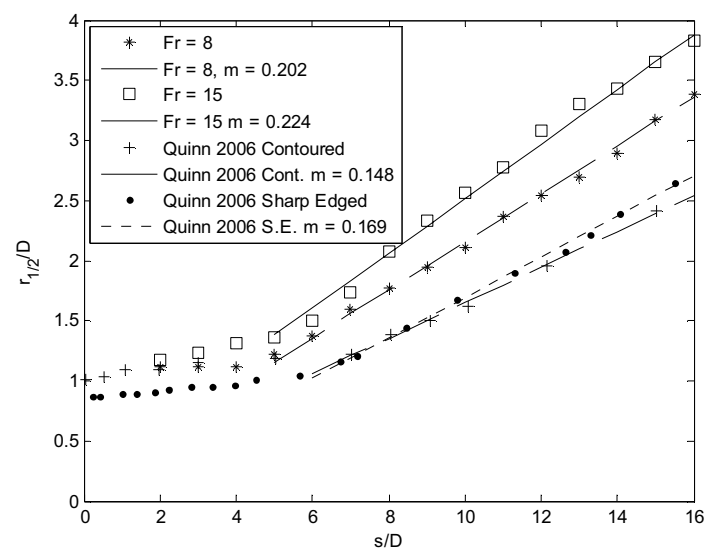

Figure 3. Widening of INBJs with $\mathrm{Re}=1000, \theta=65^{\circ}$, $\mathrm{Fr}=8$ (asterisks) and 15 (squares) and of two simple jets (Quinn 2006 [15]; sharp-edged orifice: black dots; contoured orifice: black crosses); s/D is the abscissa measured streamwise along the axis from the origin; the straight lines are the best fit ones in a least mean square sense; the coefficient $\mathrm{m}$ in the legend is the inclination of the straight lines.

Figure 4 shows the mean centerline velocity decay $\mathrm{U}_{\mathrm{C}} / \mathrm{U}_{0}$ versus the non-dimensional axial coordinate $\mathrm{s} / \mathrm{D}$ for the two simulated INBJs (colored asterisks) and for the simple jets simulated by Quinn 2006 [14], issuing from a sharp-edged (black stars) and a contoured (black rhombi) orifice. The INBJ centerline velocity decay values have a similar trend to the sharp-edged orifice ones, starting with values larger than one due to the vena contracta effect. Nonetheless, the INBJ velocity decay is higher, due to their higher widening with respect to the simple jet one. For the same reason, the obtained trend is steeper for $\mathrm{Fr}=8$ with respect to $\mathrm{Fr}=15$. Figure 4 also displays the decay of velocity computed on the profiles perpendicular to the jet axis, at an orthogonal to the axis non-dimensional distance from the axis, $r / D$, equal to the half widening of a simple jet (simple jet values used for this computations are taken from Quinn 2006 [14]) for the lower (triangles) and upper (squares) regions of the INBJs. 


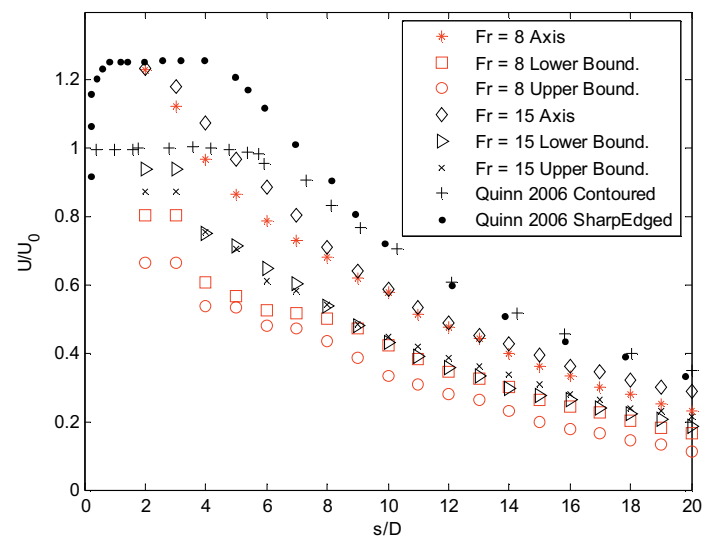

Figure 4. Streamwise non-dimensional mean velocity decay $\mathrm{U} / \mathrm{U}_{0}$ for INBJ with $\mathrm{Re}=1000, \theta=65^{\circ}$ and different $\mathrm{Fr}$ along the axis (asterisks $\mathrm{Fr}=8$, rhombi $\mathrm{Fr}=15$ ) and in the upper (circles $\mathrm{Fr}=8$, crosses $\mathrm{Fr}=15$ ) and lower (squares $\mathrm{Fr}=8$, triangles $\mathrm{Fr}=15$ ) region of the near-field, and of two simple jets (Quinn 2006 [14]; sharp-edged orifice: black dots; contoured orifice: black plus sign); the upper and lower boundary decays are measured in correspondence to the widening of a simple jet.

The highest velocities are located in the lower boundary, with a sudden decrease at around $\mathrm{s} / \mathrm{D}=4$, which corresponds to the region where the inner core of velocity tends to move toward the upper region (see Figure 2). The velocity at the upper boundary is almost constant until around $\mathrm{s} / \mathrm{D}=6$, subsequently following more gradual decay, without any step. Moreover, the velocities of the lightest INBJ $(\mathrm{Fr}=15)$ are always higher than the ones of the heaviest INBJ: this is due to the fact that these values have been measured at the same orthogonal distance from the axis and, consequently, the values of the lightest INBJ (which widens more, see Figure 3) are closer to the high velocity core than the values of the heaviest one.

In Figure 5 the Turbulent Kinetic Energy (TKE), nondimensionalised by $\mathrm{U}_{\max }{ }^{2}$, fields for the two simulated INBJs are presented; the jet axes, the same plotted in Figure 2, are superimposed as dash-dotted black lines. The plots display similar and asymmetric behavior: close to the jet origin, TKE values are low but, at few diameters from the origin, the onset of the Kelvin Helmholtz billows causes a sudden TKE increase. There are two high value regions at the jet sides, the one located at the lower boundary occurs nearer to the orifice, is shorter and is more intense with respect to the one located in the upper one which, in turn, presents lower but more persistent values. As $\mathrm{s} / \mathrm{D}$ increases, the lower TKE peak region is deflected toward the jet axis and the two peaks tend to merge into a single peak; going further, TKE tends to rapidly decrease as the INBJs become wider.

In order to better highlight the mentioned differences between the upper and lower region of INBJs, in Figure 6, the streamwise decay of $\mathrm{TKE}_{\mathrm{MAX}}$ (the maxima of TKE/ $\mathrm{U}_{\max }{ }^{2}$ computed on sections orthogonal to the jet axis) in the upper (triangles and squares) and lower (asterisks and rhombi) region of the near-field of the two INBJs with $\mathrm{Fr}=8$ (red symbols) and $\mathrm{Fr}=15$ (black symbols) and at the boundary of a simple jet (which is, of course, symmetric) with the same Re (green circles) are plotted versus s/D. All the curves have a similar trend, with an initial growth, a peak and a following decrease.
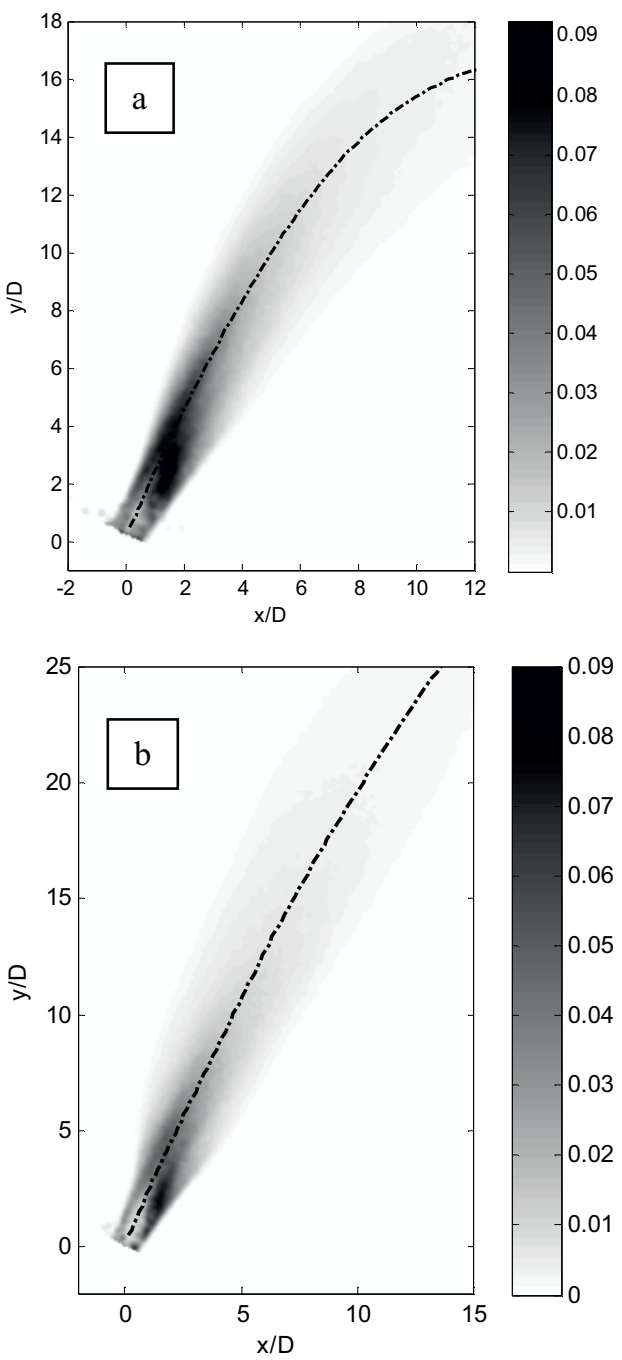

Figure 5. Map of the non-dimensional Turbulent Kinetic Energy TKE/ $\mathrm{U}_{\max }{ }^{2}$ in the near-field of an INBJ with $\operatorname{Re}=1000, \theta=65^{\circ}, \mathrm{Fr}=8$ (a) and 15 (b); the dashdotted line is the jet axis.

The values in the lower region tend to be higher than the ones in the upper region, until around $s / D=6$, where the upper region curve collapses into the lower region one. These higher values in the lower region are due to the local unstable stratification, with more intense velocity fluctuations due to the different directions of local momentum and buoyancy. The simple jet values tend to initially stay between the upper region and the lower region values, but showing a smoother trend, with a less pronounced peak, to then collapse, after around 10 diameters from the origin, to the INBJ values. 


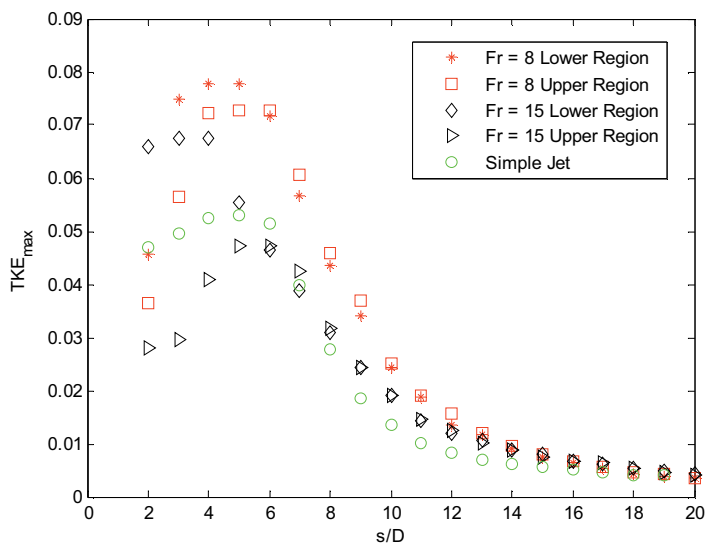

Figure 6. Streamwise decay of the maximum nondimensional Turbulent Kinetic Energy TKE/ $\mathrm{U}_{\max }{ }^{2}$ in the upper (squares $\mathrm{Fr}=8$, triangles $\mathrm{Fr}=15$ ) and lower (asterisks $\mathrm{Fr}=8$, rhombi $\mathrm{Fr}=15$ ) region of the near-field of an INBJ with $\mathrm{Re}=1000, \theta=65^{\circ}$ and different $\mathrm{Fr}$ and of a simple jet with the same $\operatorname{Re}$ (green circles).

In Figure 7 the streamwise decay of $\mathrm{TKE}_{\mathrm{INT}}$ (the integral non-dimensional Turbulent Kinetic Energy TKE/ $\mathrm{U}_{\max }{ }^{2}$ ) in the near-field of two INBJs with $\mathrm{Fr}=8$ (red asterisks) $\mathrm{Fr}=15$ (black squares) and of a simple jets with the same Re (green asterisks) is plotted along the jet axis; the integral is computed on profiles orthogonal to the jet axis, up to the half velocity jet widening above defined. The influence of $\mathrm{Fr}$ on this parameter is more evident: INBJ values start always higher than simple jet ones, with a more pronounced peak (with a higher value for the lower Fr which tends to decrease as Fr increases). The peak for the simple jet is found more streamwise if compared to the INBJ ones.

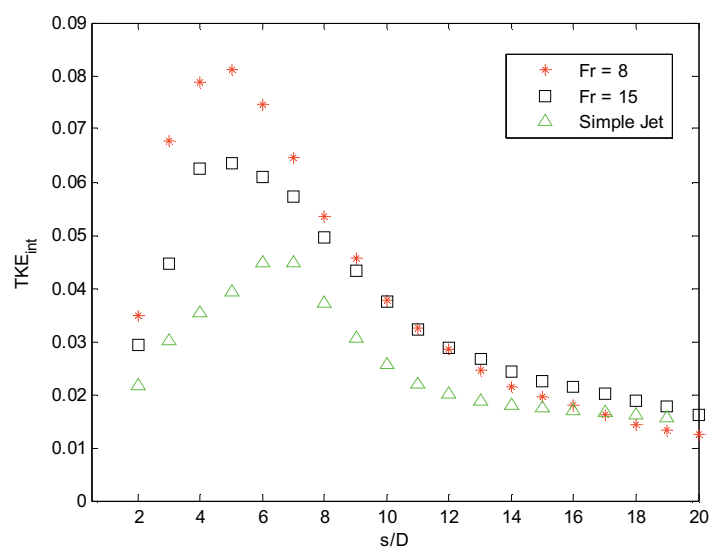

Figure 7. Streamwise decay of the integral nondimensional Turbulent Kinetic Energy TKE/ $\mathrm{U}_{\max }{ }^{2}$ in the near-field of an INBJ with $\mathrm{Re}=1000, \theta=65^{\circ}$ and different Fr (red: asterisks 8, black squares: 15) and of a simple jets with the same Re (green triangles).

In Figure $8, \mathrm{TKE}_{\mathrm{INT}}$ is separately measured at the upper (red squares $\mathrm{Fr}=8$, black triangles $\mathrm{Fr}=15$ ) and lower (red asterisks $\mathrm{Fr}=8$, black rhombi $\mathrm{Fr}=15$ ) region of the two INBJs with $\mathrm{Fr}=8$ (red) and $\mathrm{Fr}=15$ (black) and at the boundary of a simple jet (which is, of course, symmetric) with the same Re (green circles) and plotted versus s/D; differently from Figure 7 , the integrals are computed on profiles orthogonal to the jet axis, up to the half velocity jet widening, only from the axis upwards or downwards. Similar to Figure 6 considerations can be drawn, with higher values in the lower region than in the upper one that finally collapse, the simple jet values in the middle between the upper and lower region values and an asymptotic value for the simple jet similar to the INBJ ones.

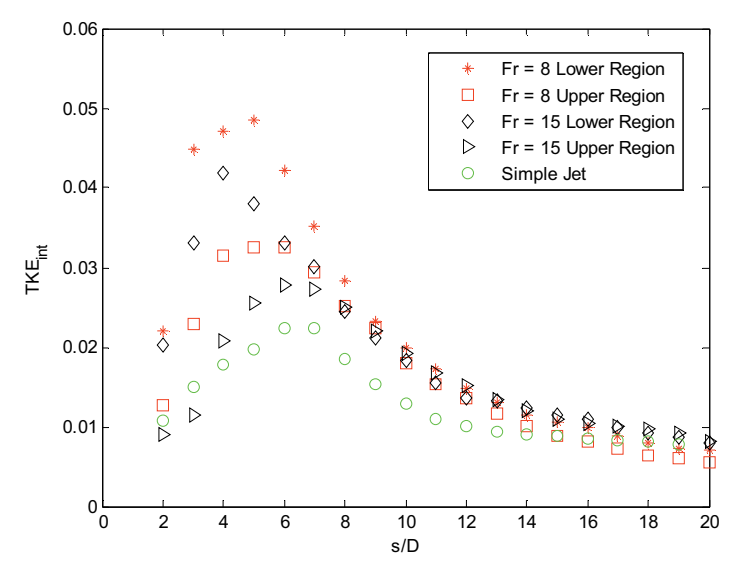

Figure 8. Streamwise decay of the integral nondimensional Turbulent Kinetic Energy TKE/ $\mathrm{U}_{\max }{ }^{2}$ in the upper (squares $\mathrm{Fr}=8$, triangles $\mathrm{Fr}=15$ ) and lower (asterisks $\mathrm{Fr}=8$, rhombi $\mathrm{Fr}=15$ ) region of the near-field of an INBJ with $\mathrm{Re}=1000, \theta=65^{\circ}$ and different $\mathrm{Fr}$ and of a simple jet with the same Re (green circles).

\section{Conclusions}

The near field behavior of inclined negatively buoyant jets, issuing from a circular sharp-edged orifice, were simulated in a laboratory model and analyzed by means of Feature Tracking Velocimetry in order to investigate the symmetry properties of this phenomenon. First and second order velocity statistics of jets with different densimetric Froude numbers were analyzed and compared to corresponding simple jet ones. Results have highlighted substantial differences between heavy and simple jet both in terms of velocity (jet widening and axial velocity decay) and Turbulent Kinetic Energy (maxima and integral values) fields. Moreover analyzed experimental data point out how asymmetry is a key feature of inclined negatively buoyant, that cannot be neglected also in the near field since it arises very close to the release point.

\section{References}

1. P.J.W. Roberts, A. Ferrier, G. Daviero, J. Hydraulic Eng. 123(8), 693-699 (1997)

2. EJ List: In: Fisher, HB - List, EJ - Koh, RCY Imberger, J - Brooks, NH (Eds): Mixing in inland 
and coastal water, New York, USA, Academic Press, (1979)

3. Besalduch, L.A., Badas, M.G., Ferrari, S., Querzoli, G., EPJ WoC, 45, art. no. 01012, (2013)

4. R.C.Y. Koh, M.S. Isaacson, N.H. Brooks, J. Hydraulic Eng., 109(2), 100-220 (1975)

5. Lindberg, W.R., Petersen, J.D: Transportation Res. Record, 1304, 219-229 (1991)

6. Hall, N., Elenany, M., Zhu, D., and Rajaratnam, N. J. Hydraul. Eng., 136(10), 727-738 (2010)

7. S. Ferrari, G. Querzoli, J. Hydraulic Research, 48(5), 632-640 (2010)

8. A. Cipollina, A. Brucato, F. Grisafi, S. Nicosia, J. Hydraulic Eng., 131(11), 1017-1022 (2005)

9. J. Mi, G.J. Nathan, D.S. Nobes, J. Fluid Eng. ASME 123(4), 878-883, (2001)

10. J. Mi, P. Kalt, G.J. Nathan, C.Y. Wong, Exp. Fluids 42(4), 625-637 (2007)

11. S.J. Wright, D.R. Wong, K.E. Zimmerman, R.B. Wallace J. Hydraulics Div. ASCE 108(HY4), 483501 (1982)

12. C. Avanzini, Proc. 4th Intl. Conf Marine Waste Water Disposal and Marine Environment \& 2nd Intl. Exhibition Materials, Equipment and Services for Coastal Waste Water Treatment Plants, Outfalls and Sealines Antalya, Turkey, Mem Ajans, Istanbul (2006)

13. C. Harris, M. Sthephens, In : Procedings of the 4th Aivey Vision Conference, pp 141-151 (1988)

14. Quinn W.R. European Journal of Mechanics B/Fluids 25 279-301(2006) 